\title{
Identification and functional Characterisation of ctr1, a Pleurotus ostreatus Gene Coding for a Copper Ttransporter
}

\author{
M. M. Peñas*; G. Azparren; A. Domínguez; \\ H. Sommer; L. Ramírez; A. G. Pisabarro \\ *Departamento de Producción Agraria, \\ Universidad Pública de Navarra, 31006 Pamplona, Spain \\ mpenas@unavarra.es
}

Copper homeostasis is primordial for life maintenance and especially relevant for ligning-degrading fungi whose phenol-oxidase enzymes depend on this micronutrient for their activity. In this paper we report the identification of a gene (ctr1), coding for a copper transporter in the white rot fungus Pleurotus ostreatus, in a cDNA library constructed from four-days old vegetative mycelium growing in submerged culture. The results presented here indicate that: (1) ctr1 functionally complements the respiratory deficiency of a yeast mutant defective in copper transport supporting the transport activity of the Ctr1 protein; (2) ctr1 transcription is detected in all P. ostreatus developmental stages (with exception of lamellae) and is negatively regulated by the presence of copper in the culture media; (3) ctr1 is a single copy gene that maps to P. ostreatus linkage group III; and (4) the regulatory sequence elements found in the promoter of ctr 1 agree with those found in other copper related genes described in other systems. These results provide the first description of a copper transporter in this white rot fungus and open the possibility of further studies on copper metabolism in higher basidiomyetes. 